\title{
MYC amplification and overexpression in primary cutaneous angiosarcoma: a fluorescence in-situ hybridization and immunohistochemical study
}

\author{
Wonwoo Shon ${ }^{1}$, William R Sukov ${ }^{1}$, Sarah M Jenkins ${ }^{2}$ and Andrew L Folpe ${ }^{1}$ \\ ${ }^{1}$ Department of Laboratory Medicine and Pathology, Mayo Clinic, Rochester, MN, USA and ${ }^{2}$ Department of \\ Biomedical Statistics and Informatics, Mayo Clinic, Rochester, MN, USA
}

MYC, a proto-oncogene located on chromosome $8 \mathrm{q} 24$, is involved in the control of cell proliferation and differentiation. Previous studies have documented high-level MYC gene amplification and MYC overexpression by immunohistochemistry $(\mathrm{IHC})$ in post-irradiation angiosarcomas, but not in primary cutaneous angiosarcoma (AS-C) or in other radiation-associated vascular proliferations, such as atypical vascular lesions. Prompted by our recent finding of MYC amplification in a primary hepatic AS, we analyzed a large number of wellcharacterized AS-C for MYC amplification and protein overexpression. Formalin-fixed, paraffin-embedded blocks from 38 AS-C were retrieved from our archives and were examined by IHC analysis and fluorescence in-situ hybridization (FISH), using a commercially available antibody and probe. For FISH analysis, the number of copies of MYC was compared with the control gene, CEN8 (MYC/CEN8 ratio). All cases occurred on sunexposed skin; no patient was known to have a history of therapeutic irradiation. Possible associations between survival and a wide variety of clinicopathological variables were evaluated using the log-rank test. By IHC analysis, MYC overexpression was present in 9/38 (24\%) AS-C (2-3+: 6 cases, $16 \%$; $1+$ : 3 cases, $8 \%$ ). By FISH analysis, $2 / 5(40 \%)$ informative cases with 2-3+ immunostaining showed high-level gene amplification. One additional case with 3+ immunostaining showed higher level aneusomy of chromosome 8 (5-8 MYC and CEN8). Two out of fourteen (14\%) IHC-negative cases also carried MYC amplification (one high level and one lower level). Low copy number gain of chromosome 8 (3-5 MYC and CEN8) was observed in AS-C with or without MYC expression. MYC amplification and MYC protein overexpression were not correlated with clinical outcome. We have shown, for the first time, MYC gene amplification and protein overexpression in primary (non-radiation-associated) AS of the skin. MYC protein overexpression in cases lacking gene amplification likely reflects other mechanisms of MYC activation. The study of a larger number of AS-C showing MYC amplification may be necessary to determine whether the behavior of such cases differs from their more common non-amplified counterparts.

Modern Pathology (2014) 27, 509-515; doi:10.1038/modpathol.2013.163; published online 4 October 2013

Keywords: angiosarcoma; cutaneous; MYC; post-irradiation

The $M Y C$ proto-oncogene, a transcription factor located on chromosome $8 \mathrm{q} 24$, is thought to be involved in the regulation of cellular proliferation, differentiation, and apoptosis. ${ }^{1}$ The mechanisms underlying $M Y C$ activation include gene amplification, activating mutations, and gene rearrangement, and appear to be different in different tumor

Correspondence: Dr AL Folpe, MD, Department of Laboratory Medicine and Pathology, Mayo Clinic, Rochester, MN, USA.

E-mail: folpe.andrew@mayo.edu

Received 11 June 2013; revised 9 July 2013; accepted 14 July 2013; published online 4 October 2013 types. ${ }^{1}$ Relatively recently, MYC amplification has been shown to be a common feature in post-irradiation and chronic lymphedema-associated angiosarcomas (secondary angiosarcomas), where it is presumed to have a key oncogenic role. ${ }^{2,3}$ In contrast, initial studies did not find $M Y C$ amplification in primary cutaneous or deep soft tissue angiosarcomas, suggesting pathogenetic differences between primary and secondary angiosarcomas. ${ }^{2-4}$ However, two relatively small series have very recently identified high-copy number $M Y C$ amplification in primary cutaneous angiosarcomas, ${ }^{5,6}$ and our own group has reported (in abstract form) $M Y C$ amplification in a 
subset of primary hepatic angiosarcomas. ${ }^{7}$ In contrast, $M Y C$ amplification seems to be absent in a distinctive group of atypical vascular lesions following irradiation ('atypical vascular lesions'), a feature that helps to distinguish them from post-irradiation angiosarcomas. ${ }^{3,8}$ At the protein level, MYC expression has been shown to be a feature of many post-irradiation angiosarcomas, but not of atypical vascular lesions or other benign cutaneous vascular proliferations.,8 Immunohistochemistry (IHC) analysis for MYC expression appears to correlate well with $M Y C$ amplification by fluorescence in-situ hybridization (FISH). ${ }^{3}$

We studied a large series of well-characterized primary cutaneous angiosarcomas for $M Y C$ amplification and MYC expression in order to more fully characterize the frequency of these events in these uncommon tumors. In addition, we correlated MYC status in these tumors with a variety of histopathological and clinical variables, including patient outcome.

\section{Materials and methods}

The Mayo Clinic Institutional Review Board granted approval for this study. We searched our institutional pathology archives for all cases coded as 'angiosarcoma' involving the skin for the period 1987-2007. Following identification of these cases, the medical records were re-reviewed and all cases thought to be post irradiation or lymphedema associated were excluded, leaving a final study population of 38 primary cutaneous angiosarcomas. Hematoxylin and eosin-stained slides were re-examined by two of the authors (WS and ALF) to confirm the original diagnosis, and a single representative tumor block was chosen for MYC immunostaining and interphase FISH analysis. The tumors were classified as 'vasoformative,' 'spindled,' 'epithelioid,' or 'mixed,' as previously described. ${ }^{9}$ Follow-up information was obtained from our medical records.

For IHC analysis, standard whole sections were immunostained for MYC (clone EP121, 1:100; Epitomics, Burlingame, CA, USA) using heat-induced epitope retrieval and the Ventana Ultraview detection system (Ventana, Oro Valley, AZ, USA). Appropriate controls were employed. Only nuclear reactivity was considered positive. All cases were scored as 'negative' ( $<5 \%$ positive), ' $1+$ ' ( $5-25 \%$ positive), ' $2+$ ', ( $26-50 \%$ positive), or ' $3+$ ' ( $\geq 51 \%$ positive).

FISH analysis for the quantitation of $M Y C$ and chromosome 8 centromere was performed on formalin-fixed, paraffin-embedded tissue sections cut at $5 \mu \mathrm{m}$ using commercially available FISH probes for MYC (8q24) labeled with Spectrum Red (Abbott Molecular, Des Plaines, IL, USA) and CEP8 (eight centromere) probe labeled with Spectrum Green (Abbott Molecular). Standard laboratory protocols and quality control measures were followed for this study. In addition to the 38 angiosarcoma specimens tested, 20 normal skin specimens without significant vascular proliferation and 22 benign vascular lesions were also evaluated as control cases. In each case, 30 interphase nuclei were analyzed in a blinded manner by two technicians (60 total nuclei). An identical protocol is used at our institution for the evaluation of Her2Neu amplification in breast carcinoma, with respect to the number of counted nuclei. The expected normal pattern for these probes is two $M Y C$ and two CEP8 signals. Amplification of the $M Y C$ locus was defined as an increased number of $M Y C$ signals and a $M Y C / C E P 8$ ratio of $\geq 2$. Polysomy was defined as a proportional gain of both $M Y C$ and CEP8 (ratio <2).

For statistical analysis, the median times to death and 5-year outcome estimates were estimated with the Kaplan-Meier method. The relationship between survival and MYC status, as well as other clinicopathological parameters (patient age, tumor size, and tumor morphology), was assessed by the

Table 1 Clinicopathological features (38 cases)

\begin{tabular}{|c|c|}
\hline \multicolumn{2}{|l|}{ Sex } \\
\hline $\mathrm{M}$ & $25(64 \%)$ \\
\hline $\mathrm{F}$ & $14(36 \%)$ \\
\hline \multicolumn{2}{|l|}{ Age at surgery (years) } \\
\hline Median & 73 \\
\hline Range & $(27-89)$ \\
\hline \multicolumn{2}{|l|}{ Site } \\
\hline Head/neck & $30(78.9 \%)$ \\
\hline Lower extremity & $4(10.5 \%)$ \\
\hline Upper extremity & $3(7.9 \%)$ \\
\hline Chest & $1(2.6 \%)$ \\
\hline \multicolumn{2}{|l|}{ Morphology } \\
\hline Vasoformative & $22(57.9 \%)$ \\
\hline Mixed & $12(31.6 \%)$ \\
\hline Spindled & $3(7.9 \%)$ \\
\hline Epithelioid & $1(2.6 \%)$ \\
\hline \multicolumn{2}{|l|}{ Local Recurrence } \\
\hline No & 7 \\
\hline Yes & 14 \\
\hline 5-year recurrence-free \% (95\% CI) & $22.2 \%(0,45.6 \%)$ \\
\hline \multicolumn{2}{|l|}{ Metastasis } \\
\hline No & 6 \\
\hline Yes & 17 \\
\hline 5-year metastasis-free \% (95\% CI) & $17.2 \%(0,37.2 \%)$ \\
\hline \multicolumn{2}{|l|}{ Death from disease } \\
\hline No & 8 \\
\hline Yes & 26 \\
\hline Death from disease & 11 \\
\hline Death from other disease & 1 \\
\hline Death from unknown cause & 14 \\
\hline 5-year survival \% (95\% CI) & $34.5 \%(18.3 \%, 50.7 \%)$ \\
\hline $\begin{array}{l}\text { Median survival, Kaplan-Meier } \\
\text { (95\% CI) }\end{array}$ & 2.7 years $(2.1-5.1$ years $)$ \\
\hline
\end{tabular}

Abbreviations: CI, confidence interval; F, female; M, male.

Note, recurrence status unknown for 17 cases, metastasis status unknown for 15 cases; final status (death/alive) unknown for 4 cases. 
Table 2 FISH and IHC results

\begin{tabular}{|c|c|c|c|c|c|}
\hline \multirow{2}{*}{ FISH result } & \multicolumn{5}{|c|}{ MYC expression by IHC (no. of cases) } \\
\hline & Negative & $1+$ & $2+$ & $3+$ & Total \\
\hline High-level amplification ( $>21$ copies $M Y C ;$ MYC:CEP8 ratio $\geqslant 2$ ) & 1 & 0 & 0 & 2 & 3 \\
\hline Low-level amplification (MYC:CEP8 ratio $\geqslant 2$ ) & 1 & 0 & 0 & 0 & 1 \\
\hline High-level polysomy chromosome 8 without $M Y C$ amplification (5-8 $M Y C$ and 5-8 CEN8) & 0 & 0 & 0 & 1 & 1 \\
\hline Low-level polysomy chromosome 8 without $M Y C$ amplification (3-5 MYC, 3-5 CEN8) & 7 & 2 & 0 & 1 & 10 \\
\hline Normal & 6 & 0 & 2 & 0 & 8 \\
\hline Total & 15 & 2 & 2 & 4 & 23 \\
\hline
\end{tabular}

Abbreviations: FISH, fluorescence in-situ hybridization; IHC, immunohistochemistry.
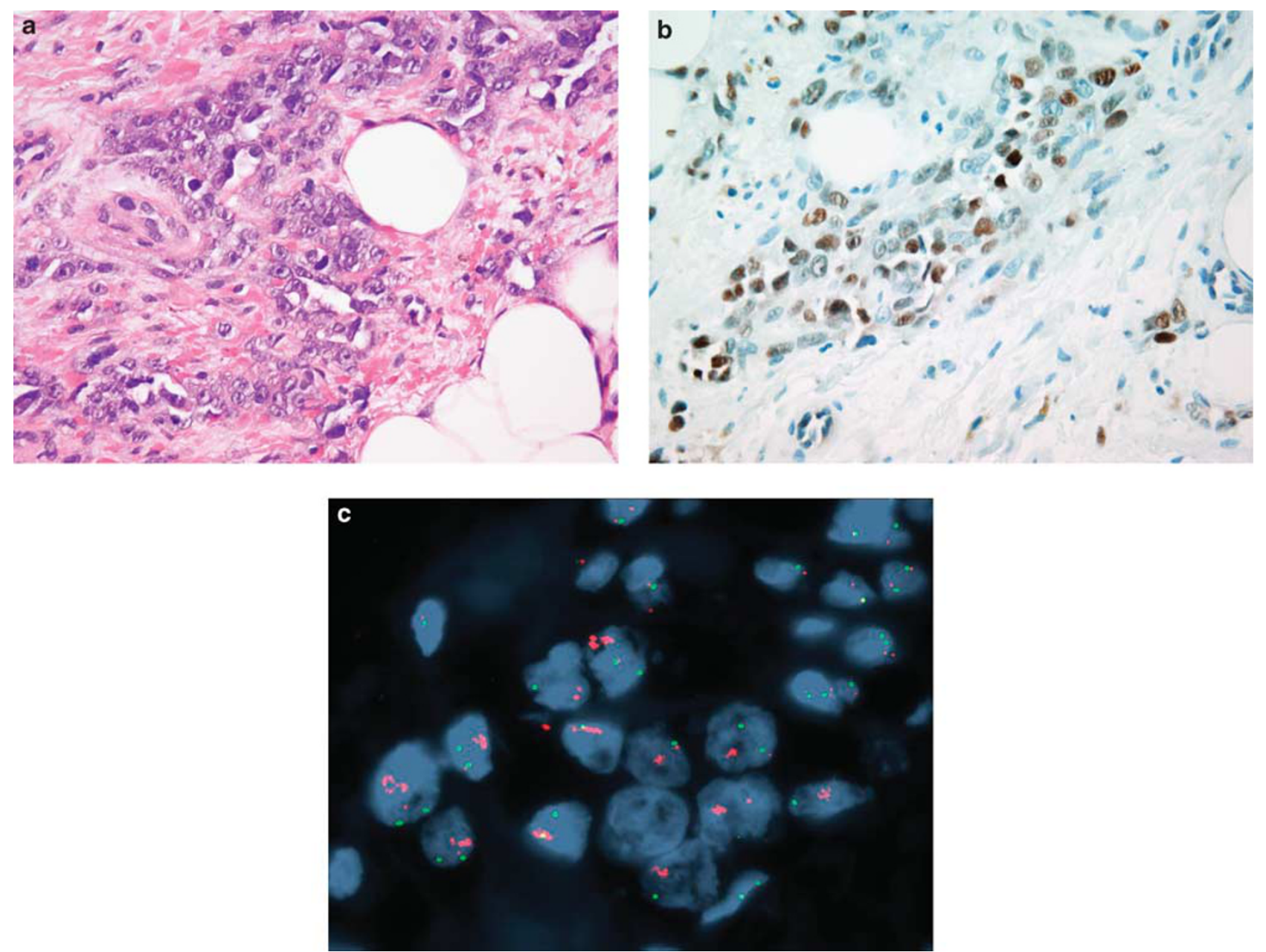

Figure 1 Primary cutaneous angiosarcoma with epithelioid morphology (a) showing nuclear MYC protein expression (b) and high-level MYC gene amplification (c); MYC: red and CEP8: green.

log-rank test. All analyses were performed in either SAS version 9 (Cary, NC, USA) or R (Foundation for Statistical Computing, Vienna, Austria).

\section{Results}

The clinicopathological findings are summarized in Table 1. The tumors occurred in 25 men and 14 women (mean age 73 years, range 27-89 years). Among the 38 with available clinicopathologic data, the tumors involved the skin of the head/neck (30 cases), leg (4 cases), arm (3 cases), and chest (1 case). The tumors ranged from 1.2 to $22 \mathrm{~cm}$ (median $7.2 \mathrm{~cm})$ and were classified as vasoformative $(22$ cases, 58\%), spindled (3 cases, 8\%), epithelioid (1 case, $3 \%$ ) and mixed (12 cases, 32\%). Figures 1-4 illustrate selected cases of angiosarcoma showing 

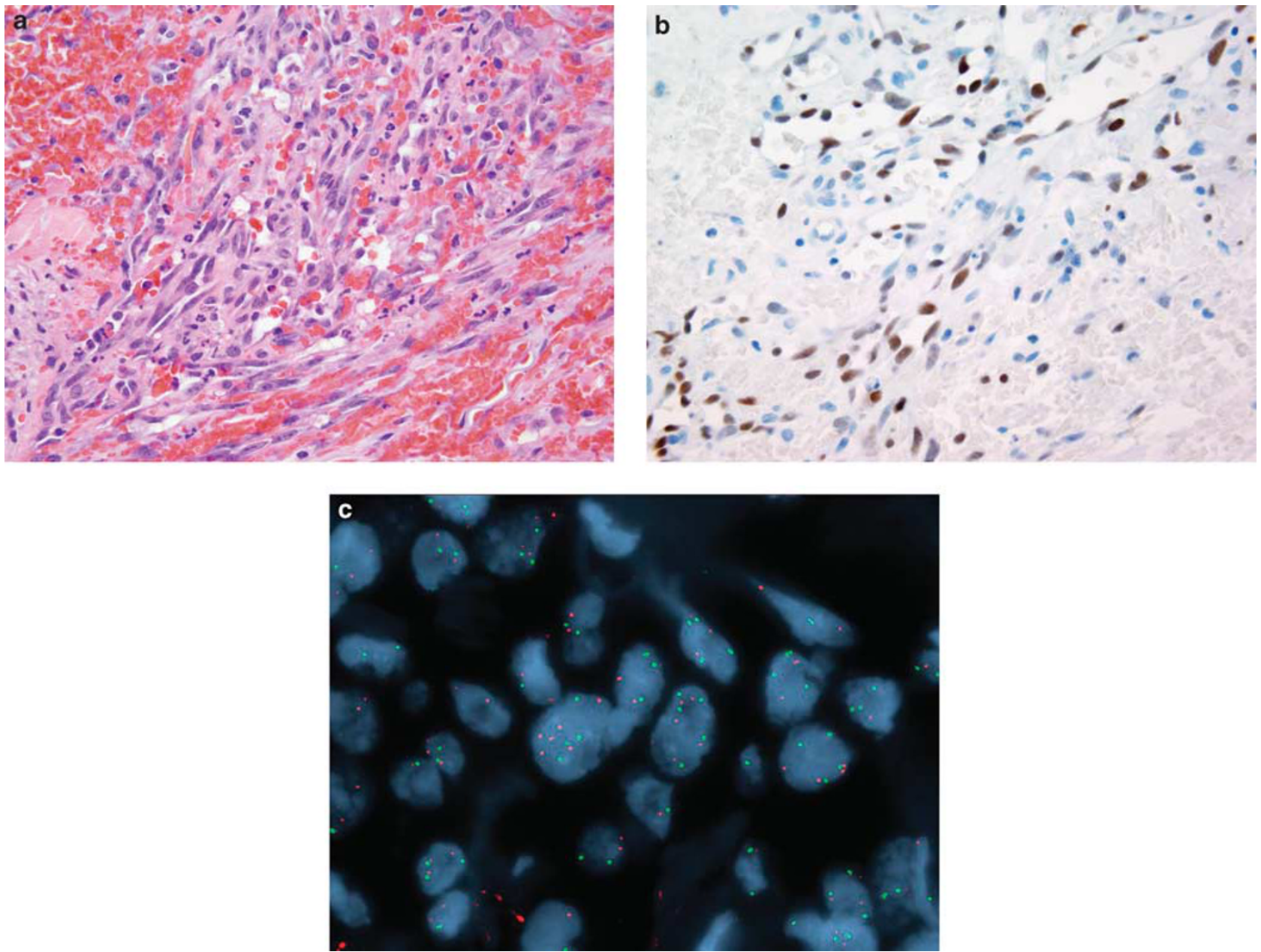

Figure 2 Another angiosarcoma (a) with strong MYC protein expression (b). By FISH, this same case showed polysomy of chromosome 8 with 5-8 red and green signals, so that the actual ratio of $M Y C$ to CEP8 is still equal or close to 1 in the tumor cells (c).

overexpression of MYC protein and/or MYC gene amplification by FISH. By IHC analysis, MYC overexpression was present in 9 of $38(24 \%)$ cases $(2-3+: 6$ cases, $16 \% ; 1+: 3$ cases, $9 \%)$, including 6 cases arising in the head/neck region, 2 from the limbs, and 1 from the chest. Surrounding non-neoplastic endothelial cells were consistently MYC negative.

Interphase FISH analysis for MYC was successfully performed on 23 primary cutaneous angiosarcomas, including 8 IHC-positive and 15 IHCnegative cases. Fourteen additional cases failed to hybridize and were not scored. The FISH and IHC analyses results are detailed in Table 2 . Of six cases that showed 2-3+ immunoreactivity, FISH analysis showed high-level amplification for MYC (>21 copies) in two cases (33\%), both from the legs. One case with $3+$ immunostaining showed polysomy of chromosome 8 without $M Y C$ amplification; the remaining three cases were found to be normal by FISH analysis. Of the 15 MYC IHC-negative cases, $2(13.3 \%)$ showed $M Y C$ amplification, including 1 case from the head/neck and 1 from the chest.
Polysomy of chromosome 8 was observed in angiosarcomas in both MYC-positive and -negative cases. Polysomic cases showed three to eight copies of MYC and CEP8. All of the control cases showed normal results by FISH analysis. The sensitivity and specificity of any positive MYC IHC (1-3+) was 66 and $70 \%$ for $M Y C$ amplification, respectively, and 47 and $66 \%$ for the presence $M Y C$ amplification or chromosome 8 copy gain, respectively. For cases showing MYC IHC of $2-3+$, the sensitivity and specificity for $M Y C$ amplification was 66 and $79 \%$, respectively, and 66 and $88 \%$ for MYC amplification or chromosome 8 copy gain, respectively.

Clinical follow-up data were available for 34 of 38 (90\%) patients (median 2.7 years, range 2 months to 19 years). Of these 34 patients, 26 died: 11 died of disease, 14 died of unknown causes, 1 died of other cause, and 8 were alive without disease. The median time to death from disease was 2.7 years (95\% confidence interval: $2.1-5.1$ years), and the 5 -year survival was $35 \%$ (95\% confidence interval: $18 \%-51 \%)$. 

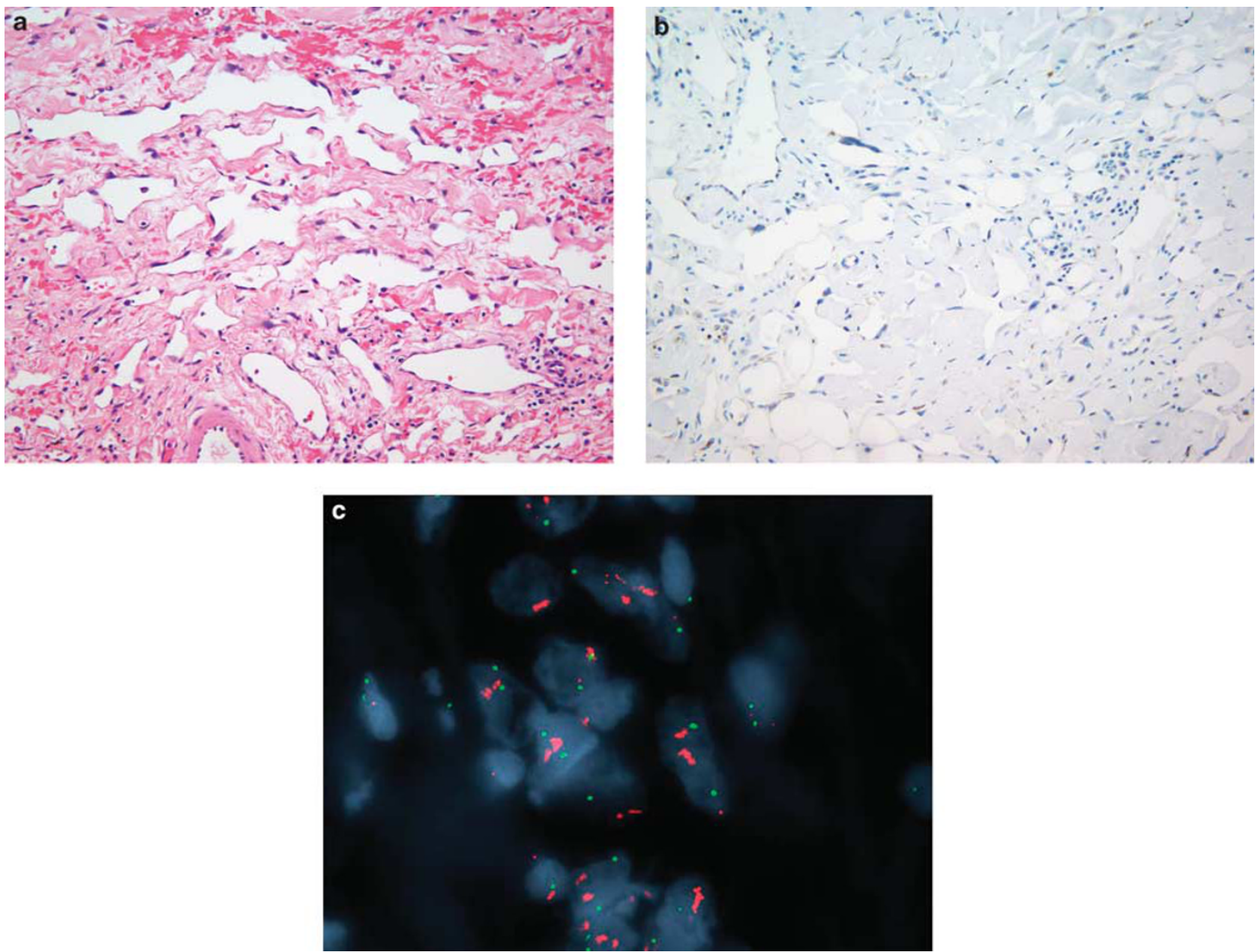

Figure 3 Angiosarcoma (a) lacking MYC protein expression (b) but showing high-level MYC gene amplification (c).

By univariate analysis, $M Y C$ amplification and MYC overexpression were not associated with overall survival (IHC, $P=0.94 ;$ FISH, $P=0.81$ ) (Figure 5). Further, none of the evaluated clinicopathological parameters was associated with survival, including patient age $(P=0.84)$, tumor size $(P=0.75)$, and tumor morphology $(P=0.53)$.

\section{Discussion}

The results of the present study, the largest to date of $M Y C$ in primary cutaneous angiosarcoma, confirm and extend upon the earlier results of Italiano et $a l^{5}$ and Hadj-Hamou et al, ${ }^{6}$ confirming the presence of both $M Y C$ amplification and MYC overexpression in a minority of such tumors. We have also identified relatively frequent chromosome 8 copy number gain without $M Y C$ amplification in a larger percentage of primary cutaneous angiosarcomas.

Regrettably, we were not able to demonstrate any relationship between $M Y C$ amplification and/or MYC overexpression, and any histopathological variables that have been previously associated with prognosis in cutaneous angiosarcoma, such as size or epithelioid versus vasoformative morphology. ${ }^{9,10}$ Similarly, we were not able to detect any difference in the clinical behavior of angiosarcomas showing MYC abnormalities versus those without this finding. Similarly, Manner et $a l^{4}$ were unable to find an association between $M Y C$ amplification and histopathological or prognosis in secondary (postirradiation) angiosarcomas. These findings are somewhat disappointing, as several prior studies (reviewed in Nesbit and Tersak ${ }^{11}$ ) have shown an association between gene amplification and/or protein overexpression of MYC and advanced stage in a variety of non-angiosarcoma human malignancies. However, we were able to detect MYC abnormalities in only a small number of primary cutaneous angiosarcomas, and it is possible that analysis of much larger series might show different results.

Somewhat unexpectedly, we did not observe a tight correlation between $M Y C$ gene amplification and protein overexpression, with only two of three high-level $M Y C$-amplified cases showing MYC overexpression and six of eight IHC-positive 

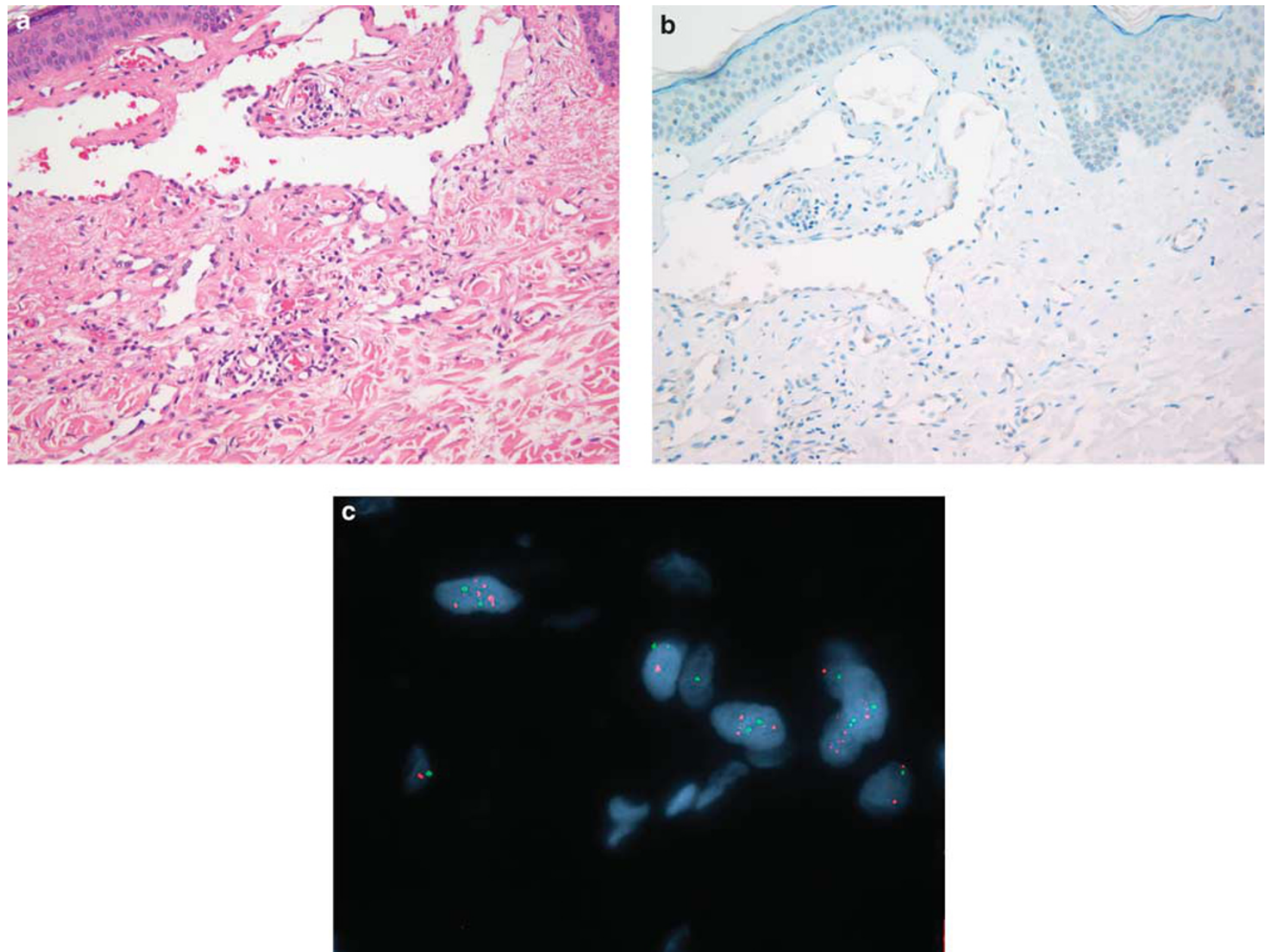

Figure 4 Angiosarcoma (a), negative for MYC expression by immunohistochemistry (b). Lower-level MYC amplification by FISH (c). Note the smaller number of red signals within the cells as compared to Figure 3.
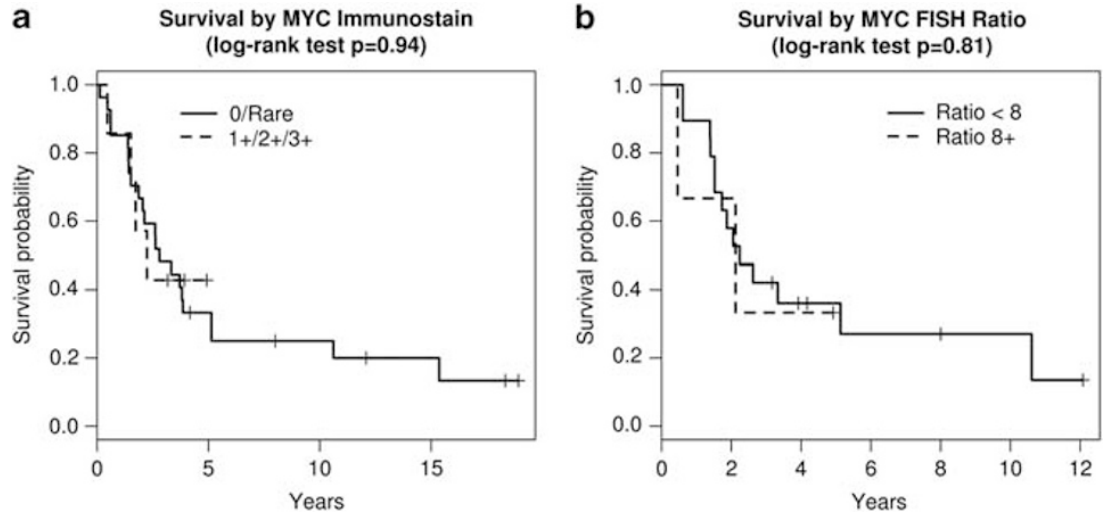

Figure 5 No differences in survival were seen in angiosarcoma patients whose tumors expressed MYC by immunohistochemistry (a) or showed high-level amplification by FISH (b) and those whose tumors did not.

cases lacking amplification by FISH analysis. The FISH-positive, IHC-negative case may be explained by limitations in the sensitivity of commercially available MYC antibodies or by the $5 \%$ threshold that we required in order to score cases as 'positive'.
This arbitrary threshold level was established, because it has been our experience in the routine evaluation of post-irradiation cutaneous vascular proliferations that rare MYC IHC-positive cells may be seen in clearly non-angiosarcoma cases. 
In addition, it is not known whether $M Y C$ amplification invariably results in MYC protein overexpression. Interestingly, four of the six IHC-positive cases lacking $M Y C$ amplification demonstrated chromosome 8 copy number gain, and it is possible that this may account for this finding. Alternatively, MYC protein expression may be regulated by other genetic or epigenetic mechanisms. For example, it is known that the neighboring gene PVT1 (8q24) is a transcriptional activator of $M Y C .^{12}$ In secondary (post-irradiation) angiosarcomas, Mentzel et $a l^{3}$ have shown MYC overexpression in all but one $M Y C$-amplified tumor.

Although the evaluation of MYC expression in other primary cutaneous vascular tumors that may enter the differential diagnosis of angiosarcoma was beyond the scope of the present study, it is difficult for us to see a potential role for these tests. This is largely because of the low frequency of $M Y C$ amplification in primary cutaneous angiosarcomas, but also a reflection of our discomfort with the lack of tight correlation between MYC FISH and IHC results. Certainly, we would be very reluctant to label a difficult primary vascular lesion as 'angiosarcoma' based only on IHC demonstration of overexpression. This is in contrast to post-irradiation lesions, where demonstration of MYC abnormalities seems to be of great value in the distinction of angiosarcoma from atypical vascular lesions. ${ }^{3}$

In summary, we have demonstrated $M Y C$ amplification and MYC protein overexpression in a subset of primary cutaneous angiosarcomas. The clinical significance of this finding is unclear, as MYC abnormalities do not seem to be related to histopathological or clinical variables. Study of larger numbers of additional cases may be necessary to determine the relative significance of gene amplification versus protein overexpression, especially as these do not always appear to be identical in all cases. In the future, identification of MYC-positive primary cutaneous angiosarcomas may prove to be of clinical significance, especially in the context of new therapeutic approaches targeting the $M Y C$ pathway. ${ }^{1,13}$

\section{Disclosure/conflict of interest}

The authors declare no conflict of interest.

\section{References}

1 Meyer N, LZ P. Reflecting on 25 years with MYC. Nat Rev Cancer 2008;8:976-990.

2 Guo T, Zhang L, Chang NE, et al. Consistent MYC and FLT4 gene amplification in radiation-induced angiosarcoma but not in other radiation-associated atypical vascular lesions. Genes Chromosomes Cancer 2011;50: 25-33.

3 Mentzel T, Schildhaus H-U, Palmedo G, et al. Postradiation cutaneous angiosarcoma after treatment of breast carcinoma is characterized by MYC amplification in contrast to atypical vascular lesions after radiotherapy and control cases: clinicopathological, immunohistochemical and molecular analysis of 66 cases. Mod Pathol 2012;25:75-85.

4 Manner J, Radlwimmer B, Hohenberger P, et al. MYC high level gene amplification is a distinctive feature of angiosarcomas after irradiation or chronic lymphedema. Am J Pathol 2010;176:34-39.

5 Italiano A, Thomas R, Breen M, et al. The miR-17-92 cluster and its target THBSI are differently expressed in angiosarcomas dependent on MYC amplification. Genes Chromosomes Cancer 2012;51:569-578.

6 Hadj-Hamou NS, Lae M, Almeida A, et al. A transcriptome signature of endothelial lymphatic cells coexists with the chronic oxidative stress signature in radiation-induced post-radiatiotherapy breast angiosarcomas. Carcinogenesis 2012;33:1399-1405.

7 Orrock JM, Mounajjed T, Zhang L, et al. Immunohistochemistry (IHC) for $\mathrm{c}-\mathrm{Myc}$ in the differential diagnosis of vascular tumors of the liver [Abstract]. Mod Pathol 2012;25(Suppl):421A.

8 Fernandez AP, Sun Y, Tubbs RR, et al. FISH for MYC amplification and anti-MYC immunohistochemistry: useful diagnostic tools in the assessment of secondary angiosarcomas and atypical vascular proliferations. J Cutan Pathol 2012;39:234-242.

9 Shon W, Jenkins SM, Ross DT, et al. Angiosarcoma: a study of 98 cases with immunohistochemical evaluation of TLE3, a recently described marker of potential taxane resposiveness. J Cutan Pathol 2011;38:961-966.

10 Deyrup AT, McKenney JK, Tighiouart M, et al. Sporadic cutaneous angiosarcomas: a proposal for risk stratification based on 69 cases. Am J Surg Pathol 2008;32:72-77.

11 Nesbit CE, Tersak JM, EV P. MYC oncogenes and human neoplastic disease. Oncogene 1999;18:3004-3016.

12 Carramusa L, Contino F, Ferro A, et al. The PVT-1 oncogene is a Myc protein target that is overexpressed in transformed cells. J Cell Physiol 2007;213:511-518.

13 Soucek L, Whitfield J, Martins CP, et al. Modeling Myc inhibition as a cancer therapy. Nature 2008;455: 679-683. 